\title{
Ernst von Metternich (1656-1727). Ein patientenzentrierter Einblick in den medizinischen Markt um 1720*
}

Kay Peter Jankrift, Annemarie Kinzelbach, Marion Maria Ruisinger

\section{Summary}

In his medical diary the physician Johann Christoph Götz from Nuremberg recorded his visits as well as his consiliary correspondence. The case of Count Ernst of Metternich who dwelled in Ratisbon and suffered from a bladder stone is particularly well documented. Thus, the source which is focusing the doctor permits to take a look at a section of the medical market managed by the patient around 1720.

Besides the Medicus ordinarius Metternich's specific network, the patient's network, comprised quite a number of local or transmigratory doctors, surgeons or lay healers as well as (former) invalids, whom he consulted in direct interviews or - in the case of Götz - by letter. The example reveals in which way the medical market was determined by the ego-network.

Analyzing this section of the market, which becomes visible through the interaction between Metternich and Götz, one can profit from Pierre Bourdieu's conception of different forms of capital. Financial capital, the exchange of goods or services against money, seems of minor importance. In lieu thereof, different cohesions become obvious, in which social, cultural and symbolical capital could become decisive for the doctor's as well as for the patient's actions and assertive for the market.

Keywords: bladder stone, consiliary correspondence, medical diary, medical market, patient history, southern Germany, 18th century

* Wir danken den Projekt-Mitarbeiterinnen Susan Splinter und Susanne Grosser für die Überlassung ihrer Transkriptions- und Forschungsergebnisse.

Prof. Dr. Kay Peter Jankrift, Deutsches Medizinhistorisches Museum, Anatomiestraße 18-20, D-85049 Ingolstadt (kay.jankrift@iek.uni-augsburg.de). Prof. Dr. Marion Maria Ruisinger, Deutsches Medizinhistorisches Museum, Anatomiestr. 18-20, D-85049 Ingolstadt (marion. ruisinger@ingolstadt.de).Dr. Annemarie Kinzelbach, Deutsches Medizinhistorisches Museum, Anatomiestraße 18-20, D-85049 Ingolstadt (kinzelbach@storicon.de). 


\section{Zusammenfassung}

Der Nürnberger Arzt Johann Christoph Götz hielt in seinem Praxisjournal sowohl seine Besuchs- als auch seine Briefpraxis fest. Besonders dicht dokumentiert ist der Fall des am Blasenstein leidenden Grafen Ernst von Metternich in Regensburg. Dadurch eröffnet die arztzentrierte Quelle den Blick auf einen patientengesteuerten Ausschnitt des medizinischen Markts um 1720.

Metternichs «Patienten-Netzwerk» umfasste neben dem Medicus ordinarius eine Fülle ortsansässiger oder auf Durchreise befindlicher Ärzte, Chirurgen, Laienheiler und (ehemaliger) Kranker, die er im direkten Gespräch oder - wie im Falle Götzes - brieflich konsultierte. Sein Beispiel veranschaulicht, wie Ego-Netzwerke den Zugang zum medizinischen Markt bestimmten.

Bei der Analyse des Marktausschnittes, der in der Interaktion von Metternich und Götz sichtbar wird, erweist sich die Unterscheidung der verschiedenen Kapitalformen im Sinne Pierre Bourdieus als hilfreich. Das pekuniäre Kapital, d.h. der Austausch von Waren bzw. Dienstleistungen gegen Geld, erscheint dabei eher zweitrangig. Stattdessen werden die unterschiedlichen Zusammenhänge deutlich, in denen soziales, kulturelles und symbolisches Kapital sowohl für den Kranken als auch für den Arzt handlungs- und damit marktbestimmend werden konnten.

Schlagworte: Blasenstein, Konsiliarkorrespondenz, Praxistagebuch, medizinischer Markt, Krankengeschichte, Süddeutschland, 18. Jahrhundert

Den 64-jährigen Grafen Ernst von Metternich (1656-1727) (Abb.1) quälte ein chronisches Blasenleiden. Auf der Suche nach Hilfe und Heilung beschränkte er sich nicht auf Gespräche mit seinem persönlichen Umfeld in Regensburg, wo er seit rund 30 Jahren - mit Unterbrechungen - als Gesandter Brandenburgs beim ständigen Reichstag wirkte, sondern nutzte auch die Möglichkeit, weiter entfernt lebende Personen brieflich zu Rate zu ziehen. Zu Metternichs Korrespondenten gehörte der Nürnberger Arzt Johann Christoph Götz (1688-1733), der die aus Regensburg empfangenen Briefe aufbewahrte und in die betreffenden Jahrgänge $(1720,1721)$ seines Praxisjournals einbinden ließ. ${ }^{1}$ Darin finden sich auch Götzes Entwürfe zu den ausführlichen Antwortschreiben, die er nach Regensburg zurücksandte. ${ }^{2}$

1 Das Praxisjournal Götzes ist Gegenstand des am Deutschen Medizinhistorischen Museum angesiedelten DFG-Projekts Ärztliche Praxis im frühen 18. Jahrhundert: Der Nürnberger Arzt Johann Christoph Götz (1688-1733).

2 Zum Phänomen der ärztlichen Konsiliarkorrespondenz in der Frühen Neuzeit vgl. zuletzt Hächler 2005; Ruisinger 2008a; Bresadola 2011, letzterer hat allerdings die deutschsprachigen Arbeiten nicht zur Kenntnis genommen. 


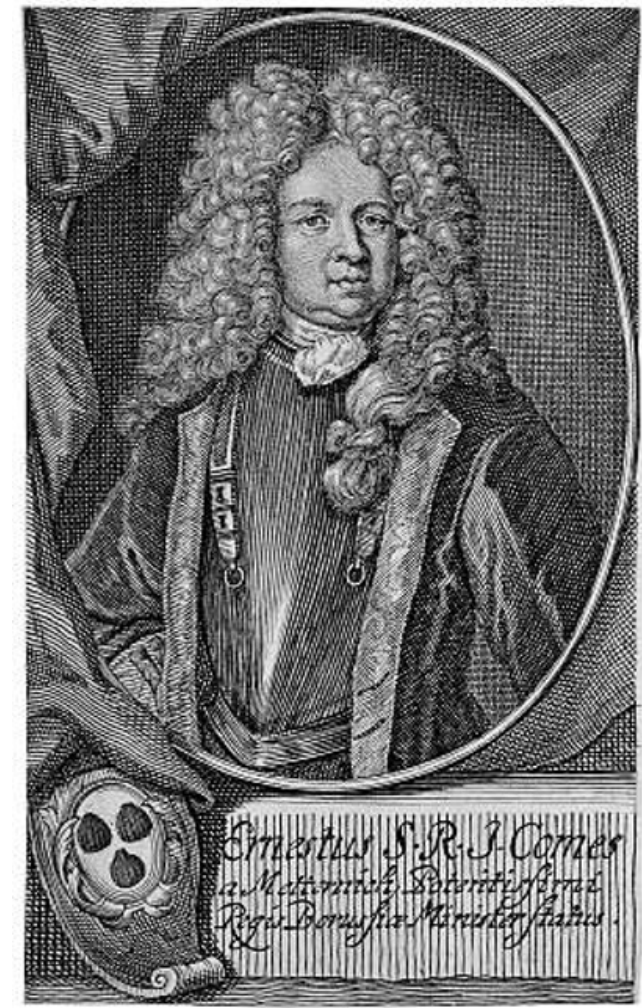

Abb. 1: Fürst Ernst von Metternich, Kupferstich $(138 \times 84 \mathrm{~mm})$ um 1700 , Johann Georg Mentzel (1677) zugeschrieben. Museen der Stadt Regensburg - Historisches Museum.
Von dem lateinischsprachigen Praxisjournal sind sieben Jahrgänge aus dem Zeitraum von 1716 bis 1726 in der Handschriftensammlung der Universitätsbibliothek Erlangen-Nürnberg erhalten. ${ }^{3}$ Götz notierte darin in kleiner, sauberer Schrift die von ihm gesehenen Kranken, seitlich vermerkte er Datum, Wochentag und Diagnose, häufig auch die beauftragte Apotheke. Ein penibel beibehaltenes Verweissystem ermöglichte es ihm, einen Krankheitsfall über mehrere Arzt-Patienten-Kontakte hinweg $\mathrm{zu}$ verfolgen. Dazwischen finden sich auch immer wieder Hinweise auf die von Götz geführte Konsiliarkorrespondenz. Offensichtlich war es für ihn unerheblich, ob die Konsultation im direkten Gespräch oder schriftlich stattfand. Beide Formen des Arzt-Patienten-Gesprächs waren selbstverständliche Bestandteile seiner ärztlichen Tätigkeit und wurden von ihm in chronologischer Folge niedergeschrieben. ${ }^{4}$

In manchen Fällen machte Götz sich sogar die Mühe, erhaltene Patientenbriefe vollständig oder auszugsweise in das Journal zu kopieren. Dadurch geben die Journalbände einen detaillierten Einblick in seine Praxis, ohne diese jedoch lückenlos oder ausschließlich abzubilden. Götz selbst charakterisierte seine Aufzeichnungen als Sammlung von Observationes et curationes Nurimbergenses ${ }^{5}$ und nahm sich damit auch die Freiheit, Beobachtungen zu notieren, die nicht in direktem Bezug zu einem Behandlungsfall standen. So enthalten die früheren Jahrgänge etwa noch tägliche meteorologische Beobachtungen; über alle Bände verteilt finden sich vereinzelte Notizen zu Obduktionen, Arzneimitteln oder Heilern.

3 UBE Ms 1200/1 (1716), 1200/2 (1717), 1200/3 (1718), 1201/1 (1720), 1201/2 (1721), 1202/1 (1725), 1202/2 (1726).

4 Zum Verhältnis von Brief- und Besuchspraxis s. Ruisinger 2008b.

5 Handschriftliche Überschrift Götzes im Journalband von 1720 (UBE Ms 1201/1, S. 1). 
Die materiell gekoppelte Dokumentation von Briefpraxis und Besuchspraxis ist eine Besonderheit der Praxisjournale Götzes. Besonders ausgeprägt findet sich dieses Phänomen in dem Jahrgangsband 1720, in dem die 474 paginierten Seiten der Praxisaufzeichnungen durch 111 im Anschluss daran eingebundene Originalbriefseiten ergänzt werden. Diese Briefe stammen von verschiedenen Autoren, handeln aber - bis auf eine Ausnahme alle vom selben Patienten: Ernst von Metternich.

Durch die Integration der Konsiliarkorrespondenz bietet das Praxisjournal somit die Möglichkeit, den medizinischen Markt um 1720 aus einer patientenzentrierten Perspektive heraus zu analysieren. Im Mittelpunkt des sichtbar werdenden Marktausschnittes steht der kranke Graf, der von Regensburg aus gut informiert und ausgesprochen selbstbewusst die Fäden zog. In der Korrespondenz mit Götz brachte Metternich vermutlich nur einen Teil der von ihm geknüpften Kontakte zur Sprache, so dass die im Folgenden versuchte Rekonstruktion des Metternich'schen Patientennetzes zwangsläufig einen fragmentarischen Charakter aufweist. Dennoch gibt diese Quelle keineswegs nur den Blick auf ein regional beschränktes Geschehen frei, das sich zwischen Regensburg und Nürnberg abspielt. Vielmehr vermitteln die Schreiben einen Eindruck davon, wie Metternich weit über die Region hinaus seine Auswahl auf dem schier unendlichen Markt an Hilfsangeboten für Blasenleidende getroffen hatte. So nimmt eine eklektisch angelegte, für den speziellen Krankheitsfall maßgeschneiderte Teilmenge des medizinischen Marktes Gestalt an, die sich über die Kommunikationsbeziehung zu Metternich definierte und von diesem aufgebaut und dirigiert wurde.

Dem Nürnberger Arzt Johann Christoph Götz kam in diesem Patientennetz lediglich eine marginale Rolle zu. Er wusste nicht, mit welchen Konkurrenten er es zu tun haben würde, und er überblickte nicht alle Angebote, die der Kranke in Anspruch nahm. Er konnte seine Position aber dadurch stärken, dass er Metternich zusätzliche Kommunikationsmöglichkeiten und Informationsquellen verschaffte, die dieser nur über ihn erreichen konnte. Dadurch machte sich Götz auch dann noch unverzichtbar, wenn seine ärztliche Hilfe bereits ausgereizt war.

Der folgende Beitrag stellt zunächst die Hauptakteure - Metternich, Götz und deren Mittelsmann Spitz - vor, um im zweiten, umfangreicheren Abschnitt das Metternich'sche Patienten-Netzwerk nachzuzeichnen, das sowohl Ärzte und Chirurgen als auch schriftliche Autoritäten, Laienheiler und andere Kranke umfasste. Zusammenfassend wird nach dem - im Sinne des Bourdieu'schen Kapitalbegriffs nicht nur pekuniär verstandenen Gewinn gefragt, den die Beteiligten aus dem Austausch von Ratschlägen, Kontakten, Geld und Arzneimitteln zogen. 


\section{Die Korrespondenten}

Am 25. Juni 1720 verfasste der Regensburger Arzt Johann Matthäus Fuchs (1693-1743) einen detaillierten lateinischen Bericht über die gesundheitlichen Beschwerden des Grafen Ernst von Metternich, der seit 1687 als brandenburgischer Gesandter am Regensburger Reichstag wirkte. ${ }^{6}$ Den Ausführungen des Heilkundigen zufolge wurde sein Patient seit über einem Jahr von einem Blasenstein gepeinigt. Dabei erstreckten sich die Schmerzen von der Lenden- zur Leistenregion, über die gesamte Schamgegend und den Damm. ${ }^{7}$ Zudem bereitete das Steinleiden große Probleme beim Wasserlassen. Fuchs hatte die körnigen Beimengungen im Harn Metternichs genau untersucht. Er stellte dabei fest, dass sich die Materie am Grund des Harnglases als kalksteinartiger Stoff niederschlug, der von einem klebrigen Schleim überzogen war. Der Sonne ausgesetzt, ließ sich diese Substanz austrocknen und zu Kugeln formen, die nach Fuchsens Befund bröckeligen Nierensteinen ähnelten. Der ärztlichen Schilderung zufolge litt der Graf darüber hinaus an hartnäckigen Unterleibskrämpfen und Stuhlzwang. Als Johann Matthäus Fuchs diesen Krankenbericht niederschrieb, hatte der brandenburgische Gesandte den Zenit seiner diplomatischen Karriere bereits überschritten.

Ernst von Metternich ${ }^{8}$ war von Kindesbeinen an auf den späteren Dienst bei Hof vorbereitet worden. ${ }^{9}$ Kaum 30 Jahre alt, wurde er als Vertreter Brandenburgs zum Immerwährenden Reichstag nach Regensburg entsandt. ${ }^{10}$ Wenig später besuchte er auch den Kurfürstenrat. Kaiser Leopold I. (1640-1705), unter dessen Herrschaft die Gegenreformation noch einmal einen Höhepunkt erlebte, erhob den Protestanten Metternich 1697 in den Grafenstand. ${ }^{11}$ Mit Leopolds Nachfolger, Joseph I. (1678-1711), verhandelte der Graf 1706 um die Königswürde für Friedrich I. von Preußen und das Fürstentum Neuburg als Teil der oranischen Erblande. Nachdem es Metternich durch sein Verhandlungsgeschick geglückt war, die Herrschaft

6 Status morbi a Dno. d. Fuchsio descriptus vom 25.6.1720 (UBE Ms 1201/1, Add. I $\delta$ ). Zu Metternichs Entsendung an den Regensburger Reichstag vgl. Friedrich 2007, 300.

7 «Dolores enim 1.) lumborum usque ad inguina sese extendentes, 2.) dolores in regione pubis, 3.) dolores in perinaeo [...]», Status morbi a Dno. d. Fuchsio descriptus vom 25.6.1720 (UBE Ms 1201/1, Add. I $\delta$ ).

8 Ernst von Metternich entstammte dem durch Johann Reinhard Freiherr von Metternich begründeten, vergleichsweise kurzlebigen Chursdorfer Familienzweig der bekannten Adelsfamilie, s. Fuchs 1994.

9 Zum Werdegang Ernst von Metternichs vgl. Friedrich 2007, 299f., sowie Reiser 1969, 140.

$10 \mathrm{Zu}$ Ernst von Metternichs Wirken auf dem Regensburger Reichstag s. Friedrich 2007 und Aretin 1997.

11 Friedrich 2007, 300. Vgl. auch Hueck 1997, 3. 
über Neuburg zu sichern, wurde er dort als Gouverneur eingesetzt und 1707 aufgrund seiner Verdienste zum königlich-preußischen Wirklichen und Geheimen Staatsrat bestellt. Im Jahre 1710 reiste Metternich als Gesandter von Wien zum Wahltag nach Frankfurt. Anschließend nahm er 1712/13 gleichfalls als Gesandter am Friedenskongress in Utrecht zur Beilegung des Spanischen Erbfolgekrieges teil. ${ }^{12}$ Seine Amtsgeschäfte in Regensburg hatte er schon zuvor mehr und mehr in die Hand seines dreizehn Jahre jüngeren Bruders Wolf (1669-1731) gelegt, der dort die brandenburgisch-bayreuthische und ansbachische Gesandtschaft versah. ${ }^{13}$ Doch wirkte Ernst von Metternich noch bis zu seinem Tod am 27. Dezember 1727 als Vertreter Brandenburgs beim Reichstag. ${ }^{14}$

Um 1720 hielt sich in Metternichs Haushalt der Lizenziat der Rechte Erhard Spitz (ca. 1685-1725) auf, ein Sohn des Altdorfer Juraprofessors Felix Spitz (1641-1717).Wie lange er schon unter dem Dach des Grafen lebte, ist unklar; möglicherweise war er als Sekretär für Metternich tätig. Der junge Rechtsgelehrte konnte ihm den Kontakt zu seinem Schwager vermitteln, der in Nürnberg als Arzt praktizierte. Seine Schwester Margaretha Spitz hatte 1713 Johann Christoph Götz geheiratet, den sie im elterlichen Professorenhaushalt in Altdorf kennengelernt hatte, wo er als Medizinstudent in Kost und Logis stand. ${ }^{15}$ Dadurch kam Spitz in der Korrespondenz des Grafen mit Götz eine Rolle zu, die weit über die eines Sekretärs hinausging. Als der vermittelnde Dritte im Bunde soll er an dieser Stelle ebenfalls mit einer biographischen Skizze vorgestellt werden - was sich allerdings deutlich schwieriger gestaltet als im Falle der beiden Hauptkorrespondenten. Die wenigen Erwähnungen seiner Person berichten lediglich, dass er an einer Gemütskrankheit gelitten habe, die um 1730 zu seinem Tod führte. ${ }^{16}$ Da Spitz sich selbst mehrmals in der Behandlung seines Schwagers befand, lassen sich diese spärlichen Informationen anhand des Praxisjournals wie folgt ergänzen:

Als Spitz im Umfeld Metternichs in Regensburg agierte, war er gut 30 Jahre alt. Götz schilderte ihn als «sanguinisch-cholerisch, ziemlich mun-

12 Granier 1954, 12.

13 Schmid 1993.

14 Zwei Wochen vor seinem Tod, am 13.12.1727, nahm Ernst von Metternich den katholischen Glauben an. Friedrich 2007, 300.

15 Splinter 2011, 6.

16 In Georg Andreas Wills Nürnbergischem Gelehrten-Lexicon (1757) steht zu lesen, dass der «sehr gelehrte» Erhard Spitz von seinem Vater in einer «gründlichen Rechtsgelehrsamkeit» angewiesen worden sei. Ihm soll jedoch eine «Liebessache [...] den Kopf verrücket haben», so dass man ihn in Nürnberg in Gewahrsam nahm, weil zu befürchten stand, dass er Hand an sich anlegen würde. «Um 1730» sei er dort, in Nürnberg, gestorben. 
ter, heiter, jedoch ein wenig ungeduldig, von hohem Wuchs, kräftig und blühend». ${ }^{17}$ Fünf Jahre später, 1725, war Spitz Hauslehrer des Prinzen Anton Ulrich von Braunschweig-Bevern (1714-1774). ${ }^{18}$ Im Juli des Jahres suchte er seinen Schwager Götz wegen Verdauungsstörungen auf, reiste dann aber nach Regensburg weiter. Die dadurch erzwungene Trennung von seiner Braut ließ ihn in ein «delirium melancholicum $»^{19}$ verfallen. Man brachte Spitz von Regensburg zurück nach Nürnberg ins Haus seiner Schwester, wo sich sein Zustand zu einem «furibundum febrem inflammatoriam»verschlechterte. Der Kranke entwickelte in seiner Raserei solche Kräfte, dass Götz ihn von einem «Eisenmeister» mit Lederriemen fixieren ließ. ${ }^{20}$ Wenige Tage später starb Spitz im Alter von etwa 40 Jahren in Nürnberg. ${ }^{21}$

Ende 1719 wandte sich Metternich durch die Vermittlung von Erhard Spitz erstmals an dessen Schwager Götz nach Nürnberg. ${ }^{22}$ Daraus entwickelte sich ein lebhafter Briefwechsel, der sich über mindestens zwei Jahre ausdehnte. Als Götz das erste Schreiben Metternichs erhielt, war er 31 Jahre alt, verheiratet und Vater von drei Kindern. Sechs Jahre zuvor war er in die Reihe der «Geschworne[n] Doctores der Arztney» aufgenommen worden, was ihn dazu berechtigte, in Nürnberg zu praktizieren. ${ }^{23} \mathrm{Zu}$ seinen Patienten zählte seit kurzem auch Karl August von Brandenburg-Kulmbach (1663-1731). ${ }^{24}$ Offensichtlich war es Götz gelungen, sich überregional einen gewissen Namen zu machen. ${ }^{25}$ Etwa zu diesem Zeitpunkt begann er, eine bemerkenswerte publizistische Tätigkeit zu entfalten. In seinen Schriften ergriff Götz in der Kontroverse zwischen der iatromechanischen Schule Friedrich Hofmanns und der animistischen Lehre Georg Stahls eindeutig Position für letzteren, den er auch zum Taufpaten seines Sohns gewählt hatte. Durch die Herausgabe der Zeitschrift Der Aufrichtige Medicus, vor allem aber durch

17 «Affini meo, aliquot ultra XXX ann., sanguineo-cholerico, alacrioris, hilarioris, impatientioris tamen aliquantulum, indolis, staturae procerae, eusarco, florido», Eintrag Götzes vom 29.2.1719 (UBE Ms 1200/3, 547).

18 «Spitz, Licentiatus Juris, pro nunc Serenissimi Principis de Bevern, Antonii Ulrici, formandis moribus et studiis vacans», Eintrag Götzes vom 30.7.1725 (UBE Ms 1202/1, 287).

19 Eintrag Götzes vom 25.8.1725 (UBE Ms 1202/1,311).

20 «ab Eisenmeister Säger vinculis e corio porcino in manu dextra et pede sinistro pavimento olligatus», Eintrag Götzes vom 31.8.1725 (UBE Ms 1202/1,336).

21 Dadurch lässt sich sein Sterbejahr von der vagen Angabe «um 1730» (Will 1757) auf 1725 präzisieren.

22 Da der Journalband des Jahres 1719 nicht erhalten ist, kann auf den Inhalt der Ende 1719 gewechselten Schreiben nur rückgeschlossen werden.

23 Splinter 2011, 6.

24 «Ann. 1719 Princeps Sereniss. Brandenburgo-Culmbacensis, Carolus Augustus, almam suam Personam Suosque curae ejus credidit», handschriftlicher Lebenslauf Götzes (LAH). - Im Praxisjournal von 1720 finden sich zahlreiche Einträge Götzes zur Behandlung Karl Augusts (UBE Ms 1201/1, 188, 207, 210, 212, 250, 301, 354, 389, 460).

25 Vgl. Splinter 2011, 6. 
seine Mitwirkung am Commercium litterarium wurde Götz zu einem bekannten Mitglied der Gelehrtenrepublik. ${ }^{26}$

Auch bei der brieflichen Beratung des vornehmen Regensburger Kranken schöpfte Götz, insbesondere in den ersten Schreiben, aus der Fülle seiner Gelehrsamkeit. Mit zahlreichen der Literatur entnommenen Therapievorschlägen und Rezepten ließ er Metternich an seinen Lesefrüchten teilhaben. Dabei zitierte er sowohl deutschsprachige, für ein interessiertes Laienpublikum verfasste Werke wie Johann Nicolaus Seitz' Trost der Armen oder Christian Weissbachs Wahrhafftige und gründliche Cur aller dem menschlichen Leibe zustossenden Kranckheiten ${ }^{27}$ als auch lateinischsprachige Fachliteratur wie Georg Ernst Stahls Theoria medica vera..$^{28}$ Aber auch Metternich und dessen medizinische Berater in Regensburg kannten die Fachliteratur, und so fand sich Götz schon bald in Erklärungsnöten: In seinem Brief vom 20. Januar hatte er kategorisch festgestellt, dass «insonderheit [...] opiata ein rechter Gifft in diesem Zustand» seien. ${ }^{29}$ Diese Behauptung stieß auf wenig Zustimmung bei Metternich, den Fuchs, «nachdem alles andere vorhero nicht helffen wollen», mit Opiaten kuriert hatte. Außerdem, so ließ man durch Spitz übermitteln, sei auch Thomas Sydenham in seinen Schriften $\mathrm{zu}$ anderen Schlüssen gekommen. ${ }^{30}$ Götz parierte die Kritik, indem er die argumentative Kraft der Fachautoren relativierte. Schließlich käme es «nicht so wohl auf die Autoritaet, als vielmehr richtig Erfahrung» an; auch Sydenham sei «ein Mensch [gewesen], der fehlen kunnte». ${ }^{31}$ Als sich Götz und Fuchs später wegen der Interpretation einer Stelle bei Stahl uneins waren, berief sich der Nürnberger auf sein Wissen um die Publikationsgepflogenheiten des ihm persönlich bekannten Autors. Dass Stahl sich in seinen Schriften zu widersprechen scheint, «ist bey Ihme nichts fremdes: dann Er nicht selten ein Medicament, so sich durch einen sonderbahren Effect bey Ihme signalisirt, an dem einem ort mit allem behörigem Lob erzählet, an einem andern aber seine Kraft wieder zweiffelhaft, und also die Leute irr, macht, daß sie Ihme nicht so gleich in die Charte gucken sollen». ${ }^{32}$

Die ausführlichen, intensiv überarbeiteten Briefentwürfe Götzes belegen, wie viel Zeit und Mühe der Nürnberger Arzt in die schriftliche Mitbehandlung des vornehmen Kranken zu investieren bereit war. Bereitwillig sandte

26 Zur Bedeutung des Aufrichtigen Medicus für das spätere Gemeinschaftsprojekt des Commercium litterarium s. Splinter 2011; zum Commercium litterarium s. Rau 2009.

27 Götz an Metternich, Entwurf vom 20.1.1720 (UBE Ms 1201/1, S. 30f.).

28 Götz an Metternich, Entwurf vom 20.1.1720 (UBE Ms 1201/1, S. 29).

29 Götz an Metternich, Entwurf vom 20.1.1720 (UBE Ms 1201/1, S. 29).

30 Spitz an Götz vom 4.2.1820, Abschrift im Journal (UBE Ms 1201/1, S. 67).

31 Götz an Spitz, Entwurf vom 10.2.1820 (UBE Ms 1201/1, 69).

32 Götz an Spitz, Entwurf vom 15.9.1720 (UBE Ms 1201/1, Add. IIIE). 
er dem Grafen das Ende 1719 erbetene ärztliche Konsil. Dieser erste Brief ist leider ebenso wenig erhalten wie Metternichs Antwort darauf. In dieser beschränkte sich der Kranke offensichtlich nicht mehr auf die Bitte um ärztlichen Beistand. Ihm war nämlich zu Ohren gekommen, dass vor einiger Zeit ein Bedienter des Kardinal Lamberg durch einen Nürnberger kuriert worden sei, und so beauftragte er Götz damit, in seinem Namen Erkundigungen zu diesem Fall einzuholen. Dies war der Beginn eines ungleichen Briefwechsels, in dem Götz in Metternichs Auftrag Fachliteratur sichtete, Erkundigungen einholte, ärztliche Gutachten verfasste und ausführliche Briefe nach Regensburg sandte. Die vom Grafen erteilten ordres gingen weit über die eigentliche ärztliche Beratungstätigkeit hinaus. Vorbehaltlos stellte der Nürnberger Arzt für Metternich die gewünschten Kontakte zu den unterschiedlichsten Anbietern auf dem medizinischen Markt her und half ihm dadurch, sein Patientennetz immer weiter $\mathrm{zu}$ spannen und dichter $\mathrm{zu}$ weben. Im folgenden Abschnitt sollen die Personengruppen vorgestellt werden, die in dieses Netz eingebunden waren.

\section{Von Metternich einbezogene Ärzte und Chirurgen}

Der Medicus ordinarius des Grafen war Johann Matthäus Fuchs, ${ }^{33}$ der zwei Jahre zuvor in Straßburg mit einer Arbeit zur Pathophysiologie der Pfortader den medizinischen Doktorgrad erworben hatte. ${ }^{34}$ Aus der Korrespondenz geht hervor, dass Metternich sich trotz gelegentlicher Sympathieschwankungen ganz wesentlich auf das Urteil von Fuchs stützte und diesen auch über alternative Diagnosen, Therapien und Empfehlungen befragte. ${ }^{35}$ Mit der Zuschreibung dieser zentralen Rolle setzte sich Metternich bei der Arztwahl über Vorurteile seiner Zeitgenossen hinweg: Der junge Arzt war nämlich ein Sohn des Regensburger Scharfrichters gleichen Namens und hatte wegen seiner Herkunft einen schweren Stand unter den Kollegen vor Ort, ${ }^{36}$ die ihm «viele Status Controversien» machten. ${ }^{37}$ Auch mit dem Rat der Stadt hatte er zu diesem Zeitpunkt deswegen «Differenzen durchzustehen». ${ }^{38}$ Einige Jahre später heiratete Fuchs die Tochter eines Augsburger Kupferstechers, Anna Catharina von und zu Steinberg auf Gallmüntz.

33 Freytag 1929, 17.

34 Fuchs 1718.

35 Spitz an Götz vom 4.2.1720, Abschrift im Journal (UBE Ms 1201/1, 67); Spitz an Götz vom 26.11.1720 (UBE Ms 1201/1, Add. XII).

36 Zur Marginalisierung von Scharfrichterfamilien siehe Nowosadtko 1994; Stuart 1999.

37 Spitz an Götz vom 4.2.1720, Abschrift im Journal (UBE Ms 1201/1, 67).

38 Fürnrohr 1965, 16. 
Offensichtlich war es ihm trotz der Widerstände gelungen, sich in Regensburg als Arzt zu etablieren und einen eigenen Hausstand zu gründen. Dennoch kam es 1735 nach dem Tode seines Vaters wieder zu einer Standeskontroverse, als der - zunächst genehmigte - feierliche mittägliche Leichenzug für den Scharfrichter wieder abgesagt und in eine wenig ehrenvolle, frühmorgendliche Beisetzung umgewandelt wurde. ${ }^{39}$

Anders als Fuchs hatte Wolfgang Matthias Brunner (1680-1722), ein weiterer Regensburger Arzt, den Metternich zwischendurch konsultierte, bereits eine längere Berufs- und Lebenserfahrung vorzuweisen. Der Sohn eines Regensburger Türmers ${ }^{40}$ promovierte 1707 in Groningen zum Dr.med. und praktizierte zunächst in Norddeutschland, wo er 1713 als Pestarzt in Hamburg selbst an der Seuche erkrankte. ${ }^{41} 1715$ veröffentlichte er in seiner Heimatstadt, in die er zwischenzeitlich zurückgekehrt war, eine Arbeit über das nichtkontagiöse Wesen der Hamburger Epidemie. ${ }^{42}$ Im Folgejahr zog Metternich ihn zu seiner Behandlung hinzu. Möglicherweise machte Brunner sich Hoffnung auf eine langfristige Bindung des prominenten Patienten, denn er widmete Metternich seine 1718 in Druck gegebene Schrift über Das in der Finsterniß scheinende aber nicht begriffene Licht. ${ }^{43}$ Bei der 1720 erfolgten Verschlechterung seines Gesundheitszustandes holte Metternich auch von Brunner wieder ein Gutachten ein. Dieser zweifelte am Vorliegen eines Blasensteins und bestätigte damit die von Metternich selbst vertretene Auffassung. Entsprechend lobend erwähnte dieser gegenüber Götz das Brunner'sche Gutachten. Doch als sich wenig später weitere gravierende Beschwerden einstellten, glaubte Spitz eine Tendenz zu erkennen, dass der Graf sich den Kritikern Brunners zugesellte, zu denen auch Götz gehörte. ${ }^{44}$

Metternich gab sich jedoch nicht mit den teilweise gegensätzlichen Ansichten der beiden Regensburger Ärzte und der schriftlichen Beratung durch Götz zufrieden. Vielmehr geht aus der Konsiliarkorrespondenz hervor, dass der Graf an den bislang erhaltenen Aussagen zu seiner Erkrankung zweifelte und nachdrücklich nach Mitteln suchte, um seine Leiden zu lindern. $\mathrm{Zu}$ diesem Zweck sammelte er Argumente weiterer Ärzte, selbst wenn diese auf sehr indirekter Information beruhten.

39 Fürnrohr 1965, 15; Dimpfelchronik (StAR), $235 f$.

40 Baader 1804.

41 Schröder 1851.

42 Brunner 1715.

43 Baader 1804.

44 Spitz an Götz, Billetnotiz vom 26.9.1720 (UBE Ms 1201/1, Add. VII); Brunner an Metternich vom 25.9.1720 (UBE Ms 1201/1, Add. VIII $\alpha$ ). Über den Meinungsumschwung Metternichs informierte Spitz seinen Schwager am 26.11.1720 (UBE Ms 1201/1, Add. XII). 
Als Metternich sich 1713 durch die in Regensburg herrschende Pest gezwungen sah, seinen Aufenthalt nach Augsburg zu verlegen, konsultierte er wegen akuter Schmerzen den dortigen Stadtphysikus und langjährigen Leopoldina-Präsidenten Lucas Schröck (1646-1730). Dieser vermutete, dass «es der Stein sei» ${ }^{45}$ - eine Diagnose, die Metternich, wie bereits erwähnt, nicht akzeptieren wollte. Möglicherweise schreckte ihn die therapeutische Konsequenz dieser Festlegung, denn gegen einen Blasenstein half - trotz aller im Umlauf befindlichen, angeblich steinzerteilenden Arzneien - letztlich nur eine lebensgefährliche Operation: der Steinschnitt. ${ }^{46}$

Die Frage, ob er einen Blasenstein hatte oder nicht, ließ Metternich keine Ruhe. In der Hoffnung, dass eine exakte Schilderung der Symptome zur Diagnoseklärung beitragen könne, übersandte er Götz einen penibel aufgesetzten Status morbi.$^{47}$ Für den Fall, dass dadurch nicht hinreichend Klarheit geschaffen werden könnte, fügte er die besorgte Frage an, «ob man solches nicht auf andere Wege gewißer exploriren könte?». Um Götz gegenüber seine Ängste zu untermauern, verwies Metternich auf den Fall eines unlängst in Regensburg verstorbenen kurbayerischen Gesandten, der «von den vornehmsten Medicis alhier tractiret» worden sei, ohne dass man sein Steinleiden erkannt hätte. Erst nach seinem Tod habe man in der Blase einen taubeneigroßen Blasenstein gefunden. ${ }^{48}$ Andererseits zitierte er den Regensburger «Medicus [...] Dr. Dietrich» ${ }^{49}$ der seinem Bruder gegenüber geäußert hatte, dass bei Metternich kein Stein vorliege, sondern dass die «Blase so dicke [sei], daß [er] nicht mehr Urin laßen könte». ${ }^{50}$

Um in der Steinfrage endlich Klarheit zu schaffen, ließ sich Metternich an einen der bekanntesten akademischen Chirurgen seiner Zeit vermitteln: Lorenz Heister (1683-1758). ${ }^{51}$ Dieser antwortete Metternich, dass er das Vorliegen eines Blasensteins zwar für wahrscheinlich halte, dass aber nur die Sondierung der Harnblase mit einem Metallkatheter Gewissheit schaffen könne. ${ }^{52}$ Leider habe er aus Metternichs Schreiben entnehmen müssen, dass es in Regensburg niemanden gäbe, «der den Catheter wohl zu gebrauchen»

45 Status morbi Metternichs an Götz als Anlage zum Brief vom 8.8.1720 (UBE Ms 1201/1, Add. I $\beta$ ) [im Folgenden zitiert als «Status morbi (UBE Ms 1201/1, Add. I $\beta$ )»].

$46 \mathrm{Zu}$ den Behandlungsmöglichkeiten des Blasensteins im frühen 18. Jh. vgl. Ruisinger 2004.

47 Status morbi (UBE Ms 1201/1, Add. I $\beta$ ).

48 Status morbi (UBE Ms 1201/1, Add. I $\beta$ ).

49 Johann G. N. Dietrichs (1681-1737) war Stadtphysikus in Regensburg und Fürstenbergischer Leibarzt; s. Schmidt-Herrling 1940, 146.

50 Metternich an Götz vom 26.11.1721 (UBE Ms 1201/2, Add. VII).

51 Lorenz Heister (1683-1758) lehrte von 1710 bis 1720 Anatomie und Chirurgie an der Nürnberger Universität in Altdorf. Im Mai 1720 folgte er einem Ruf an die Universität Helmstedt, Ruisinger 2008, 55-87.

52 Heister an Metternich vom 21.8.1720 (UBE Ms 1201/1, Add. XIII). 
wisse. Deshalb bedauerte er, dass ihn der Graf nicht schon früher konsultiert habe, da er bis vor kurzem noch in Altdorf tätig gewesen sei und von dort aus gerne Hilfe geleistet hätte. Inzwischen sei er jedoch dem Ruf an die Universität nach Helmstedt gefolgt und könne daher nur schriftlichen Rat erteilen. Die von Heister empfohlenen medikamentösen Behandlungsmethoden stimmten weitgehend mit denen des Medicus ordinarius Fuchs überein. ${ }^{53}$

Den Unterschied im Agieren des etablierten Universitätsprofessors Heister im Vergleich zum überregional noch relativ unbekannten Götz beleuchtet ein von Spitz erwähntes Postscriptum Heisters. Demzufolge verfasse dieser ein briefliches Consilium üblicherweise nur nach vorheriger Bezahlung und passe Umfang und Güte seiner Antwort an die Höhe des Honorars an. Metternich zeigte sich zwar empört über diese Forderung, ließ Heister aber die gewünschten 10 Taler und 15 Kreuzer übersenden. ${ }^{54}$

Neben ortsansässigen Ärzten und Chirurgen konsultierte der Graf auch immer wieder auswärtige, die sich nur vorübergehend im Umfeld des Immerwährenden Reichstags ${ }^{55}$ in Regensburg einfanden. ${ }^{56} \mathrm{Zu}$ diesen zählte ein Götz bekannter Dr. Gottfried Günther Schneider aus Sondershausen, der sich bei Metternichs Bruder aufhielt und mit diesem im Labor experimentierte. Spitz berichtete, dass der Graf schon seit sechs bis sieben Wochen dessen Arzneien sowie zusätzliche Frankfurter Pillen einnehme. ${ }^{57}$

Vor der Behandlung durch Schneider, im August 1720, ließ sich Metternich vierzehn Tage lang von einem «Kaiserl. Feld- u. Stabs-Chirurgus» H. Allmacher ${ }^{58}$ untersuchen, der auch zweimal einen Katheder einführte und dabei offensichtlich das Vorhandensein eines Blasensteins beweisen konnte. Zumindest begann er das Consilium, das er dem Grafen später aus Wien zuschickte, mit den Worten «Nachdem Ihro [...] Excellenz genugsam überzeiget sind, daß derselben malum eintzig und allein von einem calculo vesicae herrühre». ${ }^{59}$ Allmacher betonte die Unmöglichkeit, den Stein mit

53 Spitz an Götz vom 26.11.1720 (UBE Ms 1201/1, Add. XII).

54 Spitz an Götz vom 20.11.1720 (UBE Ms 1201/1, Add. XI). - Lorenz Heister veröffentlichte seinen Konsiliarbrief an den Grafen Jahrzehnte später in nicht anonymisierter Form in seiner Fallsammlung unter der Überschrift Von einem schweren Stein-Zufall bey einem Grafen, Heister 1753, 1002-1006.

55 Siehe Wüst 2010.

56 Heister ist der einzige chirurgisch tätige Akteur, der in der Korrespondenz namentlich genannt wird, vgl. Metternich in seinem Status morbi (UBE Ms 1201/1, Add. I $\gamma$ ); Götz an Spitz vom 15.9.1720 (UBE Ms 1201/1, Add. IIIE). Auch Brunner schrieb ganz pauschal, «der Chirurgus» sei zum Untersuchungsergebnis «Stein» gekommen, Brunner an Metternich vom 26.9.1720 (UBE Ms 1201/1, Add. VIII $\alpha$ ).

57 Spitz an Götz vom 12.11.1720 (UBE Ms 1201/1, Add. X).

58 In der Liste des Wiener Hofpersonals für die Jahre von 1715 bis 1740 wird Allmacher nicht genannt. Wir danken Monika Grass, Archivaliensammlung der Medizinischen Universität Wien, für diese Auskunft.

59 Konsilium, o. Datum (UBE Ms 1201/1, Add. III $\gamma$ ). 
Medikamenten aufzulösen, und schlug eine «gantz gelinde und kleine palliativ-Cur» vor, zu der regelmäßige Injektionen sowie ein Schlaftrunk aus warmer Milch gehörten. Beides lehnte Metternich ab: Die Injektion scheute er wegen der dafür notwendigen, schmerzhaften Katheterisierung, und von dem Schlaftrunk befürchtete er, dass die Milch und die von ihm reichlich getrunkene Limonade in seinem Magen «zu Käse» werden könnten. ${ }^{60}$

Auch Heilkundige, die sich nicht in Regensburg aufhielten, konnten für Metternich zu Hoffnungsträgern werden, wenn ihn die Nachricht von erfolgreichen Kuren erreichte. So kam ihm Ende 1719 zu Ohren, dass ein Nürnberger namens Lohner einen Bediensteten des Kardinals Lamberg mit Arzneien vom Stein befreit habe. Ohne zu zögern bediente sich der Graf der gerade erst angebahnten Verbindung zu Götz, um das Gerücht überprüfen zu lassen. Dieser folgte bereitwillig der «gegebenen Ordre» ${ }^{61}$ und wandte sich an seinen ärztlichen Kollegen Michael Friedrich Lochner von Hummelstein (1662-1720). Doch Lochner konnte sich «Zeit Lebens [keiner] gethanen Cur im Lambergischen hau $\aleph^{2}{ }^{62}$ erinnern und verwies stattdessen auf einen Nürnberger Bader gleichen Namens, vor dessen Arznei er jedoch ausdrücklich warnte. Götz schickte kurzerhand «einen guten Freund» zu dem Bader Lohner, ${ }^{63}$ der bestätigte, die Kur an dem «Lambergischen Bedienten» durchgeführt zu haben, und dem Götz'schen Boten für einen Vierteltaler sein heilendes Pulver verkaufte. Trotz eigener Bedenken übersandte Götz das fragliche «Pulver» mit dem Hinweis, er könne nicht beurteilen, ob dieses nicht vielleicht doch wirksam sei. ${ }^{64}$ Metternich, dem es inzwischen besser ging, ließ durch Schwager Spitz antworten, dass er besagtes Pulver «niemahln brauchen wollen, sondern nur aus Curiositaet [habe] kommen lassen». ${ }^{65}$

60 Spitz an Götz vom 12.9. 1720 (UBE Ms 1201/1, Add. III $\beta$ ).

61 Götz an Metternich, Entwurf vom 20.1.1720 (UBE Ms 1201/1, 27).

62 Weiter unten ist ein «Cardinal von Lamberg» erwähnt, in Frage kommen deshalb Johann Philipp Graf von Lamberg (1651 Wien - 1712 Regensburg), Bischof in Passau und Kardinal, oder dessen Neffe Joseph Dominikus Graf von Lamberg (1680 Schloss Lamberg in Steyr - 1761 Passau), Fürstbischof im Bistum Passau und ebenfalls Kardinal, www. mom-ca.uni-koeln.de/mom/DE-ABP/Urkunden/fond; www.staatliche-bibliothek-passau.de/ staadi/psn/050.html [gesehen am 15.9.2011].

63 Johann Conrad Lochner wird in den Verwaltungsakten von 1706 als seit kurzer Zeit tätiger «Bader und Wund Arzt in dem Irrer-Bad» erwähnt, der seine Lehre bei Hans Carl Krynundt, «Baders im unter Wöhr», absolviert hatte (StAN Rst N, E 5/2 Nr. 20).

64 Götz an Metternich, Entwurf vom 20.1.1720 (UBE Ms 1201/1,27-33).

65 Spitz an Götz vom 4.2.1720 (UBE Ms 1201/1, 67-70). 


\section{Von Metternich einbezogene Laienheiler und Kranke}

Für Metternich war jeder, der in dem Ruf stand, Steinkranke behandeln zu können oder selbst von einem Steinleiden genesen zu sein, ein Träger potentiell hilfreicher Informationen. Daher werden in seinen Briefen nicht nur Ärzte und Bader, sondern auch Laienheiler fassbar, d.h. Personen, die therapeutische Maßnahmen vornahmen oder Heilmittel verkauften, ohne eine einschlägige formale, obrigkeitlich anerkannte Ausbildung abgeschlossen zu haben. Zwischen diesen und den (genesenen) Kranken, die durch ihre Patientenbiographie zu Experten wurden, bestand oftmals keine eindeutige Trennung.

So ist nicht eindeutig zu erkennen, ob der in Regensburg anwesende Kardinal Christian August von Sachsen-Zeitz (1666-1725) ${ }^{66}$ sein Steinmittel aufgrund eigener oder fremder Erfahrungen empfahl. Da der in seiner Korrespondenz stets vorsichtig wirkende Metternich dem Rat des Kardinals gefolgt war, «Petram Quadratam [...] in ein kaltes Wasser mit ZitronenSafft vermischt» einzunehmen, muss der Bischof zumindest überzeugend gewirkt haben. Durch den Erfolg sah sich Metternich bestätigt, der den Abgang eines «Steinlein einer Erbsen groß» mit dem Arzneimittel in Zusammenhang brachte. ${ }^{67}$

In der Regel waren Laienheiler darauf angewiesen, dass dankbare Patienten ihre positiven Erfahrungen oder zumindest Gerüchte über Erfolge direkt und indirekt weitergaben. ${ }^{68}$ Dass dies auch für überregional mit ihren Arzneien handelnde Ärzte galt, geht aus den beiden folgenden Beispielen hervor: Im Falle eines von Heinrich Christian Kortholt aus Danzig ${ }^{69}$ vertriebenen Medikaments eilte das Gerücht dem Erfolg voraus, ${ }^{70}$ wie die von Götz übermittelten Angaben zu dem Nürnberger Steinkranken Wolfskehl zeigen: Dessen «Fräulein Schwester» gab genaue Auskunft darüber, dass ihr Bruder die Arzneien erst seit knapp fünf Tagen einnehme und dass diese

66 Christian August von Sachsen-Zeitz konvertierte 1689/91 zum Katholizismus. Er hatte Kanonikate in Regensburg (1699-1708) und schließlich in Eichstätt (1721) inne, s. Sächsische Biografie online: http://saebi.isgv.de/biografie/ChristianAugust_von_Sachsen-Zeitz_\% 281666-1725\%29 [gesehen am 15.9.2011].

67 Spitz an Götz vom 4.2.1720 (UBE Ms 1201/1, 67).

68 Vielfach gelang es Laienheilern durch die Vorlage entsprechender, meist schriftlicher, positiver Zeugnisse von (geheilten) Kranken sogar, eine obrigkeitliche Praktiziererlaubnis zu erhalten, s. Kinzelbach 1999, 177.

69 Im 18. und 19. Jh. war ein Arzt gleichen Namens bekannt, der in Kiel als Sohn eines Theologen geboren wurde und 1694 in Hamburg, später in Holland tätig war, s. DBI / 695, $433 f$. Sich auf Nachforschungen des Nürnberger Wolfskehl in Berlin beziehend, berichtet Götz von einem zweifelhaften Ruf Kortholts unter den dort lebenden Danzigern; Götz an Metternich, Entwurf vom 24.8.1720 (UBE Ms 1201/1, 324).

70 Metternich an Götz vom 8.8.1720 (UBE Ms 1201/1, Add. I $\alpha$ ). 
von Danzig her gesandten Tropfen über 30 «Ducaten» gekostet hätten und darüber hinaus noch Kosten für einen behandelnden Arzt verursachten. ${ }^{71}$ Drei Wochen später berichtete Götz, dass Wolfskehl nur sehr eingeschränkte Erfolge der Behandlung bemerkte, sich bislang keines der von Kortholt «angegebnen Phaenomenis ereignet» habe und Wolfskehl auch aufgrund der schon entstandenen $160 \mathrm{fl}$ [Gulden] Unkosten deshalb die «Medicamenta» nicht weiterempfehlen könne. Im Gespräch mit Wolfskehl hatte Götz erfahren, dass der Arzt Johann Conrad Dippel ${ }^{72}$ in den Niederlanden einen Steinkranken erfolgreich von seinem Leiden befreit habe. ${ }^{73}$ Auch diese Information leitete Götz, der den Informationshunger des gräflichen Patienten richtig einschätzte, umgehend an Metternich weiter.

Dabei griff Metternich keineswegs verzweifelt nach jedem Heilmittel, das Erfolg versprach. Vielmehr nutzte er seine Beziehungen, um Erkundigungen über den Hersteller, die Wirkung und den Erfolg sowie die Inhaltsstoffe der jeweiligen Medikamente einzuholen. Dies veranschaulicht das Beispiel des Nürnberger Büttners Schmidt, der als «Empiricus und dermahlen [...] Leib-Medicus» eines zwischen Regensburg und Wien reisenden Generals in die Korrespondenz eingeführt wird. ${ }^{74}$ Spitz teilte mit, der Nürnberger Bürger ${ }^{75}$ habe Metternich aufgesucht und versichert, dass seine «Arzneyen» selbst riesige Steine auflösen und abtreiben könnten, und habe ihm bis zum eintretenden Erfolg unentgeltlich einige Pillen und eine «kostbahre tinctur» überlassen. Zudem würden seine Arzneien auch den drohenden «Brand in der Blase» vertreiben, den er im Urin bereits erkennen könne. Trotz dieser großzügigen Versprechen verhielt sich Metternich zunächst abwartend und beauftragte Spitz, bei seinem Schwager in Nürnberg Erkundigungen einzuziehen. ${ }^{76}$ Inzwischen musste sich Schmidt einer Prüfung durch Metternichs Bruder unterziehen, der den Büttner «in der Chymie und Alchymie sehr

71 Konsiliarbrief Götzes an Metternich, Entwurf vom 24.8.1729 (UBE Ms 1201/1, 321-326). Aus dem Briefentwurf geht hervor, dass Wolfskehl nicht zu Götzes Patienten gehörte, weil dieser «HEn von Wolfskehls Medicus» erwähnt, der die Konsistenz des Urins beurteilte.

72 Johann Konrad Dippel (1673-1734) promovierte 1711 in Leiden zum Doktor der Medizin. Er war als Pietist und Alchimist bekannt, machte aber auch durch seine Inhaftierung in Altona Schlagzeilen (DBI 240 / 271-276;281).

73 Götz an Spitz vom 15.9.1720 (UBE Ms 1201/1, Add. IIIع).

74 Spitz an Götz vom 23.9.1720 (UBE Ms 1201/1, Add. V $\alpha$ ). Der General wird in der Korrespondenz als General von Berner, Bärner oder Banner genannt. Dazu würde «General Feld-Zeug-Meister Christoph von Bärner» aus Mecklenburg passen, der jedoch bereits 1711 verstorben sein soll. Siehe Zedler (1732-1750), Bd. 3, 120f.

75 Bei dem als Küfer, Büttner und Rosenbüttner bezeichneten Nürnberger Bürger Schmidt handelt es sich möglicherweise um Daniel Gottlieb Schmid, der am 22.8.1699 als Büttnermeister ins Meisterbuch eingeschrieben worden war (StAN Rst N, B 12, Nr. 1, 116). Rosenbüttner wurde er laut Götz genannt, weil er beim Rosenbad wohnte (Götz an Spitz, Entwurf vom 28.9.1720, UBE Ms 1201/1, Add. V $\beta$ ).

76 Spitz an Götz vom 23.9.1720 (UBE Ms 1201/1, Add. V $\alpha$ ). 
scharff examiniret, und von Ihm alle satisfaction erhalten» habe. Daraufhin habe Metternich beschlossen, die Pillen einzunehmen, ${ }^{77}$ ohne auf die Antwort von Götz zu warten. Dessen kurz darauf eingetroffener Brief enthielt detaillierte Mitteilungen über Schmidt, die ein zwiespältiges Urteil erkennen ließen: Der Büttner habe erst seit zwei bis drei Jahren begonnen, für sein «Brunnen-Begucken», d.h. seine Harnschau, ${ }^{78}$ bekannt zu werden; einige rühmten seine wunderbaren «Curen», andere beschuldigten ihn vieler Misserfolge. Schmidt trete zwar selbstbewusst auf und verfüge über umfangreiches, wohl angelesenes Wissen zur Medizin und Religion. Die angeblich erfolgreiche Kolik-Kur relativierte Götz allerdings aus dem Blickwinkel des Medicus des betroffenen Generals. Der approbierte Arzt, «welcher sonst dem Schmid nicht abhold sondern mit selbigem wohlbekannt ist», führe den Erfolg auf zuvor eingenommene Stahl'sche Pillen zurück, weil der General die ekelhaft schmeckende Arznei des Büttners sogleich wieder erbrochen habe. Vor allem aber berichtete Götz genauer, dass der Büttner in Nürnberg «in den sogenannten Eysen, welche der gemeine Bürger gewahrsam sind, gelegen» sei und ein Gelöbnis abgelegt habe, nicht mehr zu praktizieren. ${ }^{79}$ Darüber hinaus betonte Götz, dass Schmidt ihm persönlich keineswegs bekannt sei, auch wenn dieser das Gegenteil behaupte und ihn lobe. $\mathrm{Zu}$ den Inhaltsstoffen der Arzneien des Büttners hatte Götz gleichfalls versucht, Informationen einzuholen, die er an Metternich weitergab, indem er sogar mehrfach versprach, sich Proben und genaue Kenntnisse der Inhaltsstoffe zu beschaffen und diese nach Regensburg zu senden oder weiterzugeben. In diesem Zusammenhang wies Götz auf die Einfachheit einzelner Bestandteile hin, die er bereits zu kennen glaubte. ${ }^{80}$ Damit bewies Götz eine richtige Einschätzung Metternichs. Spitz berichtete nämlich kurze Zeit später, dass Metternich die Einnahme des Pulvers eingestellt habe, nachdem er die von Schmidt an seinen Bruder weitergegebene Rezeptur erfahren hatte: «diese schlechte Sachen [Rhabarber, Sennesblätter, Anis und Pomeranzenschale], sagt Er, können ja unmöglich helffen.» ${ }^{81}$

Im zweiten Jahr der Korrespondenz mit Götz zeigt sich Metternich gegenüber Arzneien, die ihm von Laienheilern oder ihren Patienten angepriesen wurden, ein wenig misstrauischer. Aus den vorliegenden Quellen lässt

77 Spitz an Götz vom 25.9.1720 (UBE Ms 1201/1/, Add. VI).

78 Zur Bedeutung der Harnschau im Alltag s. Stolberg 2009, v.a. 17-40, 93-130.

79 Leider fehlt im Nürnberger Staatsarchiv der Protokollband des Collegium Medicum für den fraglichen Zeitraum; auch die computergestützte Recherche in den Protokollen des Rugamt im Stadtarchiv ergab keine Informationen zu diesem Vorgang.

80 Götz an Spitz vom 28.9.1720 (UBE Ms 1201/1, Add. Vß). Später spezifizierte Götz diese zunächst vage gehaltene Andeutung in «aus Vegetabilibus», Eintrag Götzes vom 7.11. bis 9.11.1720 (UBE Ms 1201/1, 427-438).

81 Spitz an Götz vom 12.11.1720 (UBE Ms 1201/1, Add. X). 
sich allerdings nicht entnehmen, ob dem eine grundsätzliche Änderung in seiner Haltung zugrunde liegt oder ob er sich nur Götz gegenüber vorsichtiger geben wollte. Solche Vorsicht zeichnet sich im Umgang mit dem ehemaligen Wolfenbütteler «Concert-Meister» Johann Andreas Burckhardi ${ }^{82}$ ab, auf dessen angeblich großen Erfolg mit seinem steinauflösenden Medikament Götz den Grafen aufmerksam machte. ${ }^{83}$ Obwohl Götz Metternich mitteilte, dass Burckhardi ausführliche Zeugnisse auch distinguierter, erfolgreich Behandelter vorweisen könne und sich nur noch wenige Tage auf der Durchreise in Nürnberg aufhalte, äußerte sich der Graf zunächst recht zurückhaltend. Er könne diese Arzneien nur einnehmen, wenn Burckhardi die Inhaltsstoffe seiner Pillen und Pulver einem «Medico» eröffne und dieser ihm zusichern könnte, dass diese «nichts hitziges und treibendes» enthielten $\cdot{ }^{84}$ Einige Tage später bot Metternich an, dass er bereit wäre, 20 rheinische Taler zu bezahlen. Damit verband er aber zwei Bedingungen: Zum einen sollte Götz sich von Burckhardi die Bestandteile seiner Arzneien nennen lassen und sich von deren Unbedenklichkeit überzeugen; zum anderen forderte er Burckhardi auf, mit einer Anzahlung von $10 \mathrm{fl}$ als sein Gast nach Regensburg zu kommen, während er die Mittel einnehme. Stelle sich in den ersten vier Tagen der Kur kein «effect» ein, werde er die Behandlung abbrechen. Im Erfolgsfalle aber werde er ihn an «zwey andere StandesPersonen» weitervermitteln, die auch bereit seien, die geforderten $50 \mathrm{fl} \mathrm{zu}$ bezahlen. ${ }^{85}$ Da das Journal des folgenden Jahres fehlt, verfügen wir mit diesem Angebot an Burckhardi über den letzten Ausschnitt der Aktionen Metternichs, aus denen wir ersehen können, wie er seine immer wieder neu geknüpften Netzwerke geschickt nutzte, um sich auf dem für ihn nur wenig eingegrenzten Markt der Diagnosen und Kuren seines Steinleidens zurechtzufinden.

82 Von Johann Andreas Burckhardi sind in den Wolfenbütteler Musikhandschriften 32 und 33 zwei Kompositions-Manuskripte nachweisbar (Heinemann 1890, 8). Wir danken Sven Limbeck, Herzog-August-Bibliothek Wolfenbüttel, und Katja Matussek, Stadtarchiv Braunschweig, für ihre Nachforschungen.

83 Götz an Metternich, Entwurf vom 22.11.1721 (UBE Ms 1201/2,Add. VI, nach 414).Zwei Tage zuvor notierte Götz in seinem Journal ausführliche Angaben zu Burckhard, seinen Testaten und den ihm daraus bekanntgewordenen Wirkungen der steinauflösenden Mittel (UBE Ms 1201/2, 458f.).

84 Metternich an Götz vom 26.11.1721 (UBE Ms 1201/2, Add. VII, nach 414).

85 Metternich an Götz vom 1.12.1721 (UBE Ms 1201/2, Add. VIII, nach 414). 


\section{Funktionsweise des medizinischen Markts um Metternich}

Der medizinische Markt, auf dem sich Johann Christoph Götz und die beteiligten Akteure im Fall Metternich bewegen, ist von besonderer Gestalt. Diese resultiert nicht zuletzt daraus, dass der Aspekt des pekuniären Kapitals, das im Allgemeinen prägend auf das Marktgeschehen einwirkt, eine eher untergeordnete Rolle spielt. Ungleich bedeutender erscheinen auf diesem Markt kulturelles, soziales und symbolisches Kapital. ${ }^{86}$ Darüber hinaus treten in der regen Korrespondenz zwischen Johann Christoph Götz und Ernst von Metternich zwei übergeordnete Ego-Netzwerke zutage: das des Nürnberger Arztes und das des Grafen. ${ }^{87}$

Die Verflechtungen reichen weit. Sie binden bekannte, zeitgenössische Ärzte wie Georg Ernst Stahl oder Lorenz Heister ebenso ein wie mehr oder weniger illustre Laienheiler. Das gesamte Ausmaß von Götzes Netzwerk wird mit Blick auf das von ihm herausgegebene Commercium litterarium deutlich. Das soziale Kapital, aus dem der Nürnberger Arzt schöpfte, wurde von ihm in unterschiedlicher Weise eingesetzt. So verwies er in seinen Briefen an Ernst von Metternich bewusst auf seine persönliche Bekanntschaft mit Georg Ernst Stahl. Diese Verbindung, die eine Anerkennung von Götzes Wirken durch eine anerkannte Autorität suggeriert, stärkte seine Position gegenüber dem Patienten. Sein ärztlicher Rat gewann an Gewicht.

Manche Verbindungsglieder des Netzwerks traten nicht aktiv in Erscheinung, sondern waren aufgrund ihres vermeintlichen Wissens um die Behandlung von Blasensteinen lediglich indirekt in die Vorgänge dieses spezifischen Marktes eingeflochten. Darunter waren bekannte Persönlichkeiten wie der Arzt Johann Konrad Dippel (1673-1734), eine schillernde Gestalt, die zu diesem Zeitpunkt gerade wegen ihrer Inhaftierung Schlagzeilen machte, aber vorher noch in Holland einen jungen Mann namens Grunder von seinem Steinleiden erlöst haben sollte, ${ }^{88}$ oder historisch nicht greifbare Personen wie ein gewisser Chevalier de Vernon. ${ }^{89}$

Den Franzosen umgab eine Aura des Geheimnisvollen. Den Ausführungen des Briefwechsels zwischen Götz und Metternich zufolge soll er mehrere Jahre als Gefangener der Muslime in Tunis zugebracht haben. Er teilte damit

86 Bourdieu 1983, 183-198; Bourdieu 1987a, b.

87 Zur Definition von Ego-Netzwerken exemplarisch Dauser 2008, 329-347. Die Diskussionen der in den vergangenen Jahren äußerst lebhaften Netzwerkforschung können hier nicht näher behandelt werden. Zu den theoretischen Grundlagen Kalus 2011,71-103; Kalus 2010; Stegbauer/Häußling 2010; Weber 2008, 289-300; Dauser/Hächler 2008,9-30; Häberlein 2008, 315-328; Lipp 2003, 49-63. Wegbereitend für die Netzwerkforschung Reinhard 1979.

$88 \mathrm{Zu}$ Johann Konrad Dippel s. Jöcher 1750.

89 Spitz an Götz vom 25.9.1720 (UBE Ms 1201/1, Add. VI). 
das Schicksal zahlreicher Zeitgenossen, die als menschliche Ware in die Hände der berüchtigten Berber-Piraten gefallen waren..$^{90}$ Der Chevalier behauptete offenbar - so geht aus dem Briefwechsel zwischen Götz und Metternich hervor -, dass ihn sein aus der Fremde mitgebrachtes Wissen zu einer erfolgreichen Therapie des Steinleidens befähige. Hier kommt nun symbolisches Kapital zum Tragen. Das Arcanum suggerierte Wirkmächtigkeit und entzog sich gleichzeitig jeglicher Kontrolle durch den Patienten. ${ }^{91}$

Im Fall des von Götz an Ernst von Metternich vermittelten Laienheilers Johann Andreas Burckhardi kam dem Arzneimittel eine ganz andere Funktion zu. Der Graf bestand darauf, die Inhaltsstoffe der von Burckhardi zur Steinbehandlung gefertigten Pillen zu erfahren. Die Offenlegung ihrer Bestandteile sorgte für eine Enttäuschung, die den Marktwert des angepriesenen Medikaments wie auch des Heilers in den Augen Ernst von Metternichs beträchtlich senkte. Die Pillen enthielten ausschließlich Substanzen, die vergleichsweise einfach und kostengünstig zu erwerben waren. Daraufhin lehnte der adelige Patient die Einnahme der Pillen ab. Er war der Auffassung, dass seinem Leib eine Behandlung mit hochwertigeren, kostbareren Stoffen gebührte. Welche Auswirkungen diese Feststellung auf die Position Burckhardis innerhalb des übergeordneten Marktgeschehens hatte, lässt sich lediglich vermuten. Unter der Annahme, dass sich die Nachricht über die Zusammensetzung der zuvor so hochgepriesenen Pillen durch Ernst von Metternich in dessen Netzwerk verbreitete, ist davon auszugehen, dass Burckhardi dadurch an Reputation auf dem Markt einbüßte. Das Beispiel zeigt exemplarisch den schmalen Grad, auf dem sich auch Johann Christoph Götz bewegte. Enttäuschte Hoffnungen Metternichs konnten umgehend dazu führen, in Ungnade zu fallen, und damit die Tür zu Patienten aus höheren Kreisen verschließen. Um dem vorzubeugen, musste der Nürnberger Arzt in der Lage sein, ein hohes Maß an kulturellem Kapital in die Waagschale werfen zu können.

Dieses Kapital bestand vor allem aus heilkundlichem Wissen. Im Laufe seines Studiums, durch die Lektüre der zeitgenössischen wissenschaftlichen Literatur, im Dialog mit seinen ärztlichen Kollegen und nicht zuletzt durch seine Beobachtungen aus der täglichen Praxis hatte Götz sein kulturelles Kapital erworben. Der Wissenserwerb ist gekoppelt an die dafür investierte Zeit, und damit an einen Faktor, der zugleich in enger Verbindung zum pekuniären Kapital steht. Der Wert einer Arbeitsleistung ermisst sich unter anderem an der Zeit, die für diese aufgebracht werden muss. Für die An-

90 Weiss 2011. Die Geschicke eines Zeitgenossen des Chevalier de Vernon schildert jetzt Milton 2010, vgl. ferner Wilson 2009; Bono 2005.

91 Zur Bedeutung des geheimen Wissens Jütte 2011. 


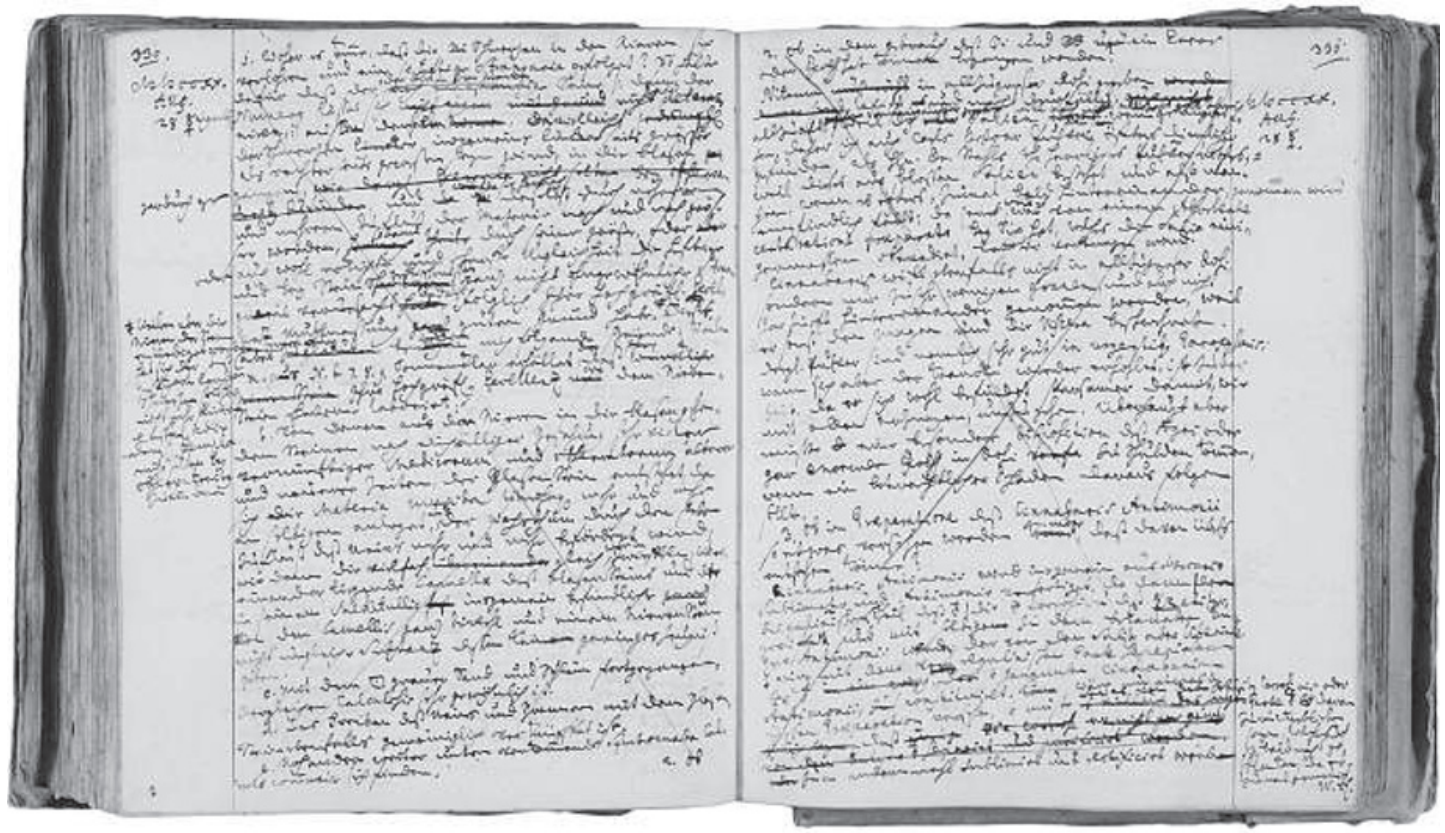

Abb. 2: Briefentwurf Götzes an Metternich vom 24.8.1720 (UBE Ms 1201/1, 322f.).

eignung seiner breiten Kenntnisse, die Götz später durch die Herausgabe des Aufrichtigen Medicus wie auch des Commercium litterarium unter Beweis stellte, musste er zweifelsohne erhebliche Zeit aufwenden. Der Arzt tritt damit sozusagen in Vorleistung. Nur wenn die spätere Entlohnung dem vorangegangenen Zeitaufwand entsprechend angemessen war, konnte dieser Vorschuss pekuniär ausgeglichen und durch einen Gewinn maximiert werden. Dass diese Rechnung nicht immer aufging, zeigt sich deutlich im Falle Metternich.

Götz verwendete viel Zeit, um dem Grafen auf seine zahlreichen Schreiben zu antworten. Die Entwürfe der Schriftstücke zeigen, wie sorgsam er jedes seiner Worte abwog (Abb. 2).

Mehrfach wird dies bei der Betrachtung von Götzes Einträgen in Bezug auf seinen berühmten Patienten im Praxisjournal deutlich. Das Exposé, das der Nürnberger Arzt darin beispielsweise am 28. August 1720 niederschrieb, enthält zahlreiche Streichungen und Randnotizen. ${ }^{92}$ Wie viele Stunden Götz damit zubrachte, eine - aus seiner eigenen Sicht zufriedenstellende Antwort für die «Hochgräfliche Excellenz» zu verfassen, lässt sich nur erahnen. Im Journal sind an diesem Tag jedenfalls keine weiteren Patientenbesuche verzeichnet. Götz scheint sich voll und ganz dem Leiden Ernst von Metternichs gewidmet zu haben. Dabei konnte er sich nie sicher sein, ob 
am Ende der Mühen auch klingende Münze fließen würde. Zwar wird in der Korrespondenz formuliert, dass eine Zahlung pekuniärer Art erwartet würde, doch drängte Götz offenbar nie auf deren Erhalt. Ob der Nürnberger Arzt je eine finanzielle Gegenleistung für seine Dienste erhalten hat, ist fraglich. Sein Zeitgenosse Lorenz Heister agierte in dieser Hinsicht weit weniger zurückhaltend. Aus seiner Reputation resultierte eine ungleich herausragendere Stellung auf dem medizinischen Markt, als sie Götz für sich in Anspruch nehmen konnte. Heister erteilte dem Grafen zwar die gewünschte Auskunft, betonte in einem Begleitschreiben jedoch, dass er die Güte des Gutachtens für gewöhnlich an die Höhe der Bezahlung anpasse.

Pekuniär zog Götz aus der Konsiliarkorrespondenz mit seinem illustren Patienten höchstens sekundär Gewinn, indem er den Fall für seine Publikationen aufbereitete. ${ }^{93}$ Wenngleich der Graf von Metternich darin nicht namentlich genannt wird, so konnten die Zeitgenossen aus den angedeuteten biografischen Details genügend Rückschlüsse auf die Identität des beschriebenen Patienten ziehen. Dieser Umstand mag sich durchaus auf die Verkaufszahlen der von Götz herausgegebenen Schriften ausgewirkt haben. Offensichtlich wird in dem Briefwechsel zwischen dem Nürnberger Arzt und Ernst von Metternich aber auch, dass für Götz nicht die pekuniäre Entlohnung, sondern die Hoffnung auf Zuwachs an sozialem wie symbolischem Kapital im Vordergrund stand und sein geduldiges Agieren maßgeblich bestimmte. So bringt er seinen Wunsch zum Ausdruck, durch Metternichs Fürsprache «einen caracter oder professionem» zu erhalten.${ }^{94}$ Doch auch auf diesen Lohn hoffte er anscheinend vergeblich.

Das symbolische Kapital, das ihm der rege Kontakt mit dem Grafen von Metternich einbrachte, suchte Götz indes bewusst zu nutzen. Am Ende seines Lebenslaufs, den er seinem Antrag um Aufnahme in die Leopoldina beifügte, nutzte er seine prominentesten Patienten geradezu als Aushängeschilder seiner ärztlichen Fähigkeiten. Durch den Verweis auf seine ärztliche Beziehung zu Ernst von Metternich und Karl August von Brandenburg-Kulmbach sucht der Nürnberger Arzt sich so gut wie möglich in der Gelehrtenrepublik zu positionieren.

93 Götz publizierte den Fall Metternich 1726 und 1727 in der von ihm herausgegebenen Zeitschrift Der Aufrichtige Medicus (Nr. 8, 31-32; Nr. 9, 33-35; Nr. 10, 37-40; Nr. 11, 41-44; Nr. 12, 45-48; Nr. 13, 49-52; Nr. 14, 53-60, Nr. 17, 65-68). 94 Spitz an Götz vom 30.10.1720 (UBE Ms 1201/1, Add. IX). 


\section{Archivsiglen}

$\begin{array}{ll}\text { LAH } & \text { Archiv der Deutschen Akademie der Naturforscher Leopoldina, Halle } \\ \text { StaAN } & \text { Staatsarchiv Nürnberg } \\ \text { StAN } & \text { Stadtarchiv Nürnberg } \\ \text { StAR } & \text { Stadtarchiv Regensburg } \\ \text { UBE Ms } & \text { Universitätsbibliothek Erlangen-Nürnberg, Handschriftenabteilung }\end{array}$

\section{Bibliographie}

Aretin, Karl Otmar von, Das Alte Reich 1648-1806, Bd. 2: Kaisertradition und österreichische Großmachtpolitik (1648-1745) (Stuttgart 1997)

August, Ritter von, «Spitz, Felix», Allgemeine Deutsche Biographie 35 (1893), 216f.

Baader, Klement Alois, Eintrag «Wolfgang Matthias Brunner», Das gelehrte Baiern. Bd.1 (1804), zit. nach DBI / 153, 292

Bono, Salvatore, Lumi e corsari: Europa e Maghreb nel Settecento (Perugia 2005)

Bourdieu, Pierre, «Ökonomisches Kapital, kulturelles Kapital, soziales Kapital», in: Kreckel, Reinhard (Hrsg.), Soziale Ungleichheiten (Göttingen 1983) 183-198

Bourdieu, Pierre, Die feinen Unterschiede. Kritik der gesellschaftlichen Urteilskraft (BadenBaden 1987a)

Bourdieu, Pierre, Sozialer Sinn. Kritik der theoretischen Vernunft (Frankfurt a.M. 1987b)

Bresadola, Marco, «A Physician and a Man of Science: Patients, Physicians, and Diseases in Marcello Malpighi's Medical Practice», BHM 85 (2011) 193-221

Brunner,Wolfgang Matthias, Merkwürdige Observationes bei der sogenannten Contagion, welche sich 1712 in Hamburg angefangen - durch welche bewiesen wird, daß sowohl das Contagium derselben Ursach nicht sei, als auch die dagegen gemachte politische Anstalten überflüssig etc. (Regensburg 1715)

Dauser, Regina, «Qualitative und quantitative Analyse eines Ego-Netzwerks am Beispiel der Korrespondenz Hans Fuggers (1531-1598)», in: Regina Dauser/Stefan Hächler u.a. (Hrsg.), Wissen im Netz. Botanik und Pflanzentransfer in europäischen Korrespondentennetzwerken des 18. Jahrhunderts, Colloquia Augustana 24 (Berlin 2008) 329-347

DBI, Deutscher Biographischer Index, Microfiche-Ausgabe (München 1986)

Freytag, Rudolf, «Verzeichnis der Regensburger Aerzte (bis 1850)», Regensburger Anzeiger 246 (1929) [Sonderdruck mit eigener Paginierung]

Friedrich, Susanne, Drehscheibe Regensburg. Das Informations- und Kommunikationssystem des Immerwährenden Reichstags um 1700, Colloquia Augustana 23 (Berlin 2007)

Fuchs, Johann Matthäus, Dissertatio Inauguralis Medica De Affectibus, Cum Vena Portae Connexionem habentibus. Diss. med. (Straßburg 1718)

Fuchs, Peter, «Metternich», Neue Deutsche Biographie, Bd. 17 (Berlin 1994), 232-235

Fürnrohr, Otto, «Scharfrichter in der Reichsstadt Regensburg». Blätter des Bayerischen Landesvereins für Familienkunde 28 (1965) 14-16

Granier, Gerhard, Der Deutsche Reichstag während des Spanischen Erbfolgekrieges (1700-1714) (Bonn 1954)

Häberlein, Mark, «Netzwerkanalyse und historische Elitenforschung. Probleme, Erfahrungen und Ergebnisse am Beispiel der Reichsstadt Augsburg», in: Regina Dauser/Stefan Hächler u.a.(Hrsg.), Wissen im Netz. Botanik und Pflanzentransfer in europäischen Korrespondentennetzwerken des 18. Jahrhunderts Colloquia Augustana 24 (Berlin 2008) 315-328

Hächler, Stefan, «Arzt aus Distanz. Fernkonsultationen bei Albrecht von Haller», in: Martin Stuber/Stefan Hächler/Luc Lienhard (Hrsg.), Hallers Netz. Ein europäischer Gelehrtenbriefwechsel zur Zeit der Aufklärung (Basel 2005) 317-349

Heinemann, Otto von, Die Handschriften der Herzoglichen Bibliothek zu Wolfenbüttel (Wolfenbüttel 1890) 
Heister, Lorenz, Medizinische, Chirurgische und Anatomische Wahrnehmungen, Bd. 1 (Rostock 1753)

Hueck, Walter von, Adelslexikon, Genealogisches Handbuch des Adels 116, Bd. 9 (Limburg a. d. Lahn 1997)

Jöcher, Christian Gottlieb, Eintrag «Johann Konrad Dippel», Allgemeines Gelehrten-Lexicon, Bd. 2 (1750), zit. nach DBI / 240, 271-276

Jütte, Daniel, Das Zeitalter des Geheimnisses. Juden, Christen und die Ökonomie des Geheimen (1400-1800) (Göttingen 2011)

Kalus, Maximilian, «Kaufmannsnetzwerke im europäisch-asiatischen Handel am Ende des 16. Jahrhunderts», in: Rolf Walter (Hrsg.), Globalisierung der Geschichte (Stuttgart 2011) 71-103

Kalus, Maximilian, Pfeffer-Kupfer-Nachrichten. Kaufmannsnetzwerke und Handelsstrukturen im europäisch-asiatischen Handel am Ende des 16. Jahrhunderts (Augsburg 2010)

Kinzelbach, Annemarie, «Konstruktion und konkretes Handeln: Heilkundige Frauen im oberdeutschen Raum, 1450-1700», in: Historische Anthropologie 7 (1999) 165-190

Lipp, Carola, «Struktur, Interaktion, räumliche Muster. Netzwerkanalyse als analytische Methode und Darstellungsmittel sozialer Komplexität», in: Silke Götsch (Hrsg.), Komplexe Welt. Kulturelle Ordnungssysteme als Orientierung. 33. Kongress der Deutschen Gesellschaft für Volkskunde in Jena 2001 (Münster 2003) 49-63

Milton, Giles, Weißes Gold. Die außergewöhnliche Geschichte von Thomas Pellow und das Schicksal weißer Sklaven in Nordafrika (Stuttgart 2010)

Nowosadtko, Jutta, Scharfrichter und Abdecker. Der Alltag zweier «unehrlicher Berufe» in der Frühen Neuzeit (Paderborn u.a. 1994)

Rau, Tilman T. R., Die erste medizinische Wochenschrift in Deutschland und die Anfänge des medizinischen Journalismus (Bremen 2009)

Reinhard, Wolfgang, Freunde und Kreaturen. «Verflechtung» als Konzept zur Erfassung historischer Führungsgruppen, römische Oligarchie um 1600 (München 1979)

Reiser, Rudolf, Adeliges Stadtleben im Barockzeitalter. Internationales Gesandtenleben auf dem Immerwährenden Reichstag zu Regensburg (München 1969)

Ruisinger, Marion Maria, «Der vierte Weg zum Stein. Die Lithotomie nach 〈Frère Jacques» als Goldstandard bei Johann Jacob Rau und Lorenz Heister», in: Vorstand der Internationalen Nitze-Leiter-Forschungsgesellschaft für Endoskopie (Hrsg.), Lithotomie versus Lithotripsie. Historisch und Rezent, Schriften der Internationalen Nitze-Leiter-Forschungsgesellschaft für Endoskopie, Bd. 4 (Wien 2004) 37-45

Ruisinger, Marion Maria, Patientenwege. Die Konsiliarkorrespondenz Lorenz Heisters (16831758) in der Briefsammlung Trew, Medizin, Gesellschaft und Geschichte, Beihefte 28 (Stuttgart 2008a)

Ruisinger, Marion Maria: «Briefpraxis versus Besuchspraxis. Das Beispiel Lorenz Heister (1683-1758)», in: Elisabeth Dietrich-Daum/Martin Dinges u.a. (Hrsg.), Arztpraxen im Vergleich: 18.-20. Jahrhundert. Veröffentlichungen des Südtiroler Landesarchivs, Bd. 26 (Innsbruck u.a. 2008b) 65-77

Schmid, Josef Johannes, «Metternich, Wolf von», in: Biographisch-Bibliographisches Kirchenlexikon, Bd. 5 (Nordhausen 1993), Sp. 1399

Schmidt-Herrling, Eleonore: Die Briefsammlung des Nürnberger Arztes Christoph Jacob Trew (1695-1769) in der Universitätsbibliothek Erlangen (Erlangen 1940)

Schröder: Eintrag «Wolfgang Matthias Brunner», Lexikon der hamburgischen Schriftsteller. Bd. 1 (1851), zit. nach DBI / 153, 294

Splinter, Susan: «Der Aufrichtige Medicus, eine Zeitschrift des Nürnberger Arztes Johann Christoph Götz (1688-1733) als Vorläufer des Commercium Litterarium», in: Jahrbuch für Kommunikationsgeschichte 13 (2011), 5-15

Stegbauer, Christian/Roger Häußling (Hrsg.), Handbuch Netzwerkforschung (Wiesbaden 2010)

Stolberg, Michael, Die Harnschau. Eine Kultur- und Alltagsgeschichte (Köln u.a. 2009)

Stuart, Kathy, Defiled trades and social outcasts; honour and ritual pollution in early modern Germany (Cambridge u.a. 1999)

Weber, Wolfgang E. J., «Pikante Verhältnisse. Verflechtung und Netzwerk in der jüngeren historisch-kulturwissenschaftlichen Forschung», in: Regina Dauser/Stefan Hächler u.a. (Hrsg.), Wissen im Netz. Botanik und Pflanzentransfer in europäischen Korrespondentennetzwerken des 18. Jahrhunderts, Colloquia Augustana 24 (Berlin 2008) 289-300 
Weiss, Gillian Lee, Captives and corsairs. France and slavery in the early modern Mediterranean (Stanford, Calif. 2011)

Will, Georg Andreas, Nürnbergisches Gelehrten-Lexicon. Bd. 3 (1757), zit. nach DBI / 1203, 355

Wilson, Peter Lamborn, Piraten, Anarchisten, Utopisten: Mit ihnen ist kein Staat zu machen (Berlin 2009)

Wittwer, Philipp Ludwig: Entwurf einer Geschichte des Kollegiums der Aerzte in der Reichsstadt Nürnberg (Nürnberg 1792)

Wüst, Wolfgang: «Methodische Impulse? Regensburg als Schnittstelle zwischen Reichs- und Landesgeschichte», in: Appl,Tobias (Hrsg.), Regensburg, Bayern und das Reich (Regensburg 2010) 247-268

Zedler,Johan Heinrich: Großes vollständiges Universal-Lexikon [...].(Halle,Leipzig 1732-1750) 